\title{
Screening for Alcohol Use in Pregnancy: a Review of Current Practices and Perspectives
}

\author{
Danijela Dozet ${ }^{1,2} \cdot$ Larry Burd $^{3} \cdot$ Svetlana Popova ${ }^{1,2,4,5}$
}

Accepted: 13 September 2021 / Published online: 22 September 2021

(C) The Author(s), under exclusive licence to Springer Science+Business Media, LLC, part of Springer Nature 2021

\begin{abstract}
Global trends of increasing alcohol consumption among women of childbearing age, social acceptability of women's alcohol use, as well as recent changes in alcohol use patterns due to the COVID-19 pandemic may put many pregnancies at higher risk for prenatal alcohol exposure (PAE), which can cause fetal alcohol spectrum disorder (FASD). Therefore, screening of pregnant women for alcohol use has become more important than ever and should be a public health priority. This narrative review presents the state of the science on various existing prenatal alcohol use screening strategies, including the clinical utility of validated alcohol use screening instruments. It also discusses barriers for alcohol use screening in pregnancy, such as practitioner constraints, unplanned pregnancies, delayed access to prenatal care, and stigma associated with substance use in pregnancy, providing recommendations to address these barriers. By implementing consistent alcohol use screening, prenatal care providers have the opportunity to facilitate access to counseling and brief interventions and thus, to prevent new cases of FASD and improve maternal and child health.
\end{abstract}

Keywords Alcohol $\cdot$ Pregnancy $\cdot$ Screening $\cdot$ Fetal alcohol spectrum disorder $\cdot$ Fetal alcohol syndrome $\cdot$ Prevention

\section{Abbreviations}

ASSIST Alcohol, Smoking, and Substance Involvement Screening Test

AUD Alcohol use disorder

Svetlana Popova

lana.popova@camh.ca

1 Institute for Mental Health Policy Research, Centre for Addiction and Mental Health, 33 Ursula Franklin Street, Toronto, ON M5S 2S1, Canada

2 Institute of Medical Science, Faculty of Medicine, University of Toronto, Medical Sciences Building, 1 King's College Circle, Toronto, ON M5S 1A8, Canada

3 Department of Pediatrics, School of Medicine and Health Sciences, Pediatric Therapy Services, Altru Health System, North Dakota Fetal Alcohol Syndrome Center, University of North Dakota, Grand Forks, ND 58202, USA

4 Dalla Lana School of Public Health, University of Toronto, 155 College Street, Toronto, ON M5T 3M7, Canada

5 Factor-Inwentash Faculty of Social Work, University of Toronto, 246 Bloor Street W, Toronto, ON M5S 1V4, Canada 


$\begin{array}{ll}\text { AUDIT } & \text { Alcohol Use Disorders Identification Test } \\ \text { CAGE } & \text { Cut down, Annoyed, Guilty, Eye-opener } \\ \text { FAS } & \text { Fetal alcohol syndrome } \\ \text { FASD } & \text { Fetal alcohol spectrum disorder } \\ \text { HSQ } & \text { Hospital Screening Questionnaire } \\ \text { NET } & \text { Normal Drinker, Eye Opener, Tolerance } \\ \text { PAE } & \text { Prenatal alcohol exposure } \\ \text { PCAP } & \text { Parent Child Assistance Program } \\ \text { PIP } & \text { Pregnancy Information Program } \\ \text { SES } & \text { Socioeconomic status } \\ \text { SMAST } & \text { Short Michigan Alcohol Screening Test } \\ \text { SUD } & \text { Substance use disorder } \\ \text { SURP-P } & \text { Substance use risk profile in pregnancy } \\ \text { T-ACE } & \text { Tolerance, Annoyance, Cut Down, Eye-opener } \\ \text { TQDH } & \text { Ten Question Drinking History } \\ \text { TWEAK } & \text { Tolerance, Worried, Eye-opener, Amnesia, K/Cut Down) } \\ \text { WHO } & \text { World Health Organization } \\ \text { WIC } & \text { Women, Infant and Child program }\end{array}$

The levels of alcohol use among women of childbearing age (15-49 years) are currently increasing in most countries globally, due to a number of factors such as economic development, changing gender roles, and social acceptability of alcohol use in women (WHO, 2018). The COVID-19 pandemic has additionally impacted alcohol use patterns among both genders globally (Rehm et al, 2020), and the emerging evidence from some countries suggests that the pandemic can exacerbate patterns of alcohol use, which can, in turn, increase alcohol-attributable problems (Pollard et al., 2020; Rehm et al, 2020). In the USA, for example, there were significant past-year increases in drinking frequency (14\%), number of heavy drinking occasions (41\%), and alcohol-related problems (39\%) among women in 2020 (Pollard et al., 2020).

Globally, about $10 \%$ of women in the general population consume alcohol during pregnancy (Popova et al., 2017a), though meta-analyses indicate this is much higher in some countries: over $60 \%$ of women in the general population of Ireland consume alcohol during pregnancy, $47 \%$ in Belarus, $46 \%$ in Denmark, $41 \%$ in the UK, and 37\% in Russia (Popova et al., 2017a). Certain sub-populations of women across countries may be at higher risk of alcohol use during pregnancy compared to the general population, such as women with substance use disorders (SUDs), women with fetal alcohol spectrum disorder (FASD), women of lower socioeconomic status (SES), and women from marginalized cultural groups (Fraser et al., 2012; McQuire et al., 2020; Montag, 2016; Popova et al., 2017b). For example, the prevalence of alcohol use among pregnant Inuit women in northern Quebec, Canada, was over 6 times higher as compared to that observed in the general population of Canada (60.5\% vs 10\%, respectively) (Fraser et al., 2012). Among women who consumed alcohol in pregnancy in the UK, binge drinking was reported to be more common among women of lower socioeconomic status (SES) (Scholder et al., 2014).

It is well-documented that alcohol use during pregnancy is associated with numerous adverse pregnancy and neonatal outcomes, including stillbirth, spontaneous abortion, low birthweight, preterm birth, intrauterine growth retardation, small for gestational age (Lebel et al., 2011; Henriksen et al., 2004; Nykjaer et al., 2014; O'Connor et al., 2002; Patra et al., 
2011), and FASD (Jones et al., 1973a; Jones et al., 1973b; Sokol et al., 2003; May et al., 2013). Even relatively low levels of PAE can negatively affect the developing fetus and increase the risk for FASD (Chambers et al., 2019; May et al., 2013). It is estimated that 1 in 13 infants with PAE will develop FASD, and there are 1726 new FASD cases per day, globally (Burd \& Popova, 2019). The economic costs of PAE and FASD are substantial in any society, due to the numerous comorbid conditions that are common in individuals with FASD (Oh et al, 2020; Popova et al., 2016a, 2016b) as well as typical adverse social outcomes (e.g., increased involvement in the child welfare system) (Popova et al., 2019; Brownell et al., 2019). In Canada alone, FASD poses an annual cost burden of \$1.3-2.3 billion (Popova et al., 2013), and globally, the annual costs of care per child and adult with FASD are \$22,810 and \$24,308, respectively (Greenmyer et al., 2018).

Prevention at time of pregnancy is only one of several key global health FASD prevention initiatives. It has been estimated that preventing one case of FASD incurs only $3 \%$ of the costs it would require to provide support services to individuals with FASD (Greenmyer et al., 2019), and this is related to the high service utilization of individuals with FASD. With the elimination of alcohol use during pregnancy, maternal and neonatal health outcomes can be improved, and new cases of FASD can be prevented. This places screening for PAE at the forefront of FASD prevention, as it demonstrates the potential to reduce the cost and service burden in healthcare and service systems globally.

Recent global trends, including increased alcohol consumption among women of childbearing age (WHO, 2018), a high proportion of unplanned pregnancies (Bearak et al., 2018), and the COVID-19 pandemic (Sher, 2020), may put many pregnancies at even higher risk for unintentional PAE and FASD. Therefore, the detection and prevention of PAE is of the utmost importance in improving maternal and child health outcomes and preventing new cases of FASD. During the span of each pregnancy, prenatal care providers can implement alcohol screening practices and instruments in their clinical practice to identify PAE and reduce associated harms. Screening for PAE is an example of an effective population-based strategy to reduce the harmful use of alcohol globally (WHO, 2014a). The purpose of this narrative review was to provide an overview of existing prenatal alcohol use screening strategies as well as to describe the clinical utility of validated alcohol use screening instruments and identify barriers for their implementation. Between May 2020 and December 2020, the following databases were searched: PubMed, Web of Science, MEDLINE, EMBASE, and CINAHL Plus. Twenty-three screening strategies were identified, which can be divided into 3 levels: (1) clinician-directed questions in prenatal care encounters, (2) structured, validated alcohol use screening instruments, and (3) laboratory-based tools and biomarkers.

\section{Screening Strategies}

\section{Level 1: Clinician-Directed Questions in Prenatal Care Encounters}

The first level of screening refers to the routine questions about alcohol use that are asked by the provider during the prenatal care encounters(s), which may occur at one or multiple timepoints during the pregnancy. Prenatal care providers can screen for alcohol use that occurred prior to pregnancy recognition and mitigate maternal stress by providing necessary counseling on the importance of protective factors (e.g., continued alcohol abstention, proper nutrition), for the remainder of the pregnancy. 
Globally, only an estimated 1-3\% of women drinking during pregnancy are identified by healthcare providers as doing so (Burd \& Popova, 2019). Research suggests there are also some inconsistencies in the capture of this information based on provider types and countries. For example, among a range of health professionals in Australia, only 45\% reported routinely asking pregnant women about their alcohol use (Payne et al., 2005). This is consistent with the findings in the USA, which indicate that $38 \%$ of medical doctors and residents reported consistently surveying pregnant women about their alcohol use (Arnold et al., 2013). A study of midwives in Norway, however, found that $97 \%$ reported asking women about alcohol use at the first prenatal care encounter (Wangberg et al., 2015). Similarly, a national study of midwifery practices in Switzerland found that questions were posed to pregnant women about alcohol use routinely by midwives ranging from 81 to $85 \%$ of the time, depending on the specific region (Lemola et al., 2020).

The first level of screening by healthcare professionals using standard questions to assess alcohol use during pregnancy is paramount to understanding which women would require further screening with a validated alcohol use screening tool, as the progression to this step stems from the initial maternal self-report (Sarkar et al., 2010). The identification of women at risk of alcohol-exposed pregnancies and children with FASD, therefore, may be missed if the first level of alcohol use screening is not implemented consistently and thoroughly. Notably, for FASD prevention, it is important for all prenatal care providers to be well-informed about any levels of alcohol consumption of pregnant women in their care, and not simply risky levels of drinking that are commonly assessed in screening instruments (Poole et al., 2019).

\section{Level 2: Validated Alcohol Use Screening Instruments}

Table 1 presents 14 instruments, which can be used to screen for alcohol use in pregnant women, 9 of which focus solely on alcohol use screening, while the other 5 screen for substances in addition to alcohol.

Only 5 of these tools have been specifically developed and/or validated for alcohol use screening in pregnant women. These 5 tools are the T-ACE (Tolerance, Annoyance, Cut Down, Eye-opener), TWEAK (Tolerance, Worried, Eye-opener, Amnesia, K/Cut Down), Alcohol Use Disorders Identification Test-consumption subset (AUDIT-C), the 4P's Plus, and the 1-Question Screen (WHO, 2014b; Poole et al., 2019; Chasnoff et al., 2005; Williams et al., 2013; Montag, 2016). Based on individualized assessment needs, prenatal care providers may choose to screen for other substances in pregnancy as well, which can be done using the 4Ps Plus, ASSIST (Alcohol, Smoking and Substance Involvement Screening Test), HSQ (Hospital Screening Questionnaire), PIP (Pregnancy Information Program), and SURP-P (Substance Use Risk Profile in Pregnancy) screening instruments.

The T-ACE is a four-item screening tool and was the first sensitive screen for risk drinking that was developed and validated for use in pregnant women screened in obstetricgynecologic practice and assesses risk drinking, which was initially defined as the consumption of one or more ounces of alcohol per day while pregnant (Sokol et al., 1989). Both paper-based questionnaires and clinician-directed T-ACE question formats have been found to be acceptable and effective in screening (Chang et al., 2001). Notably, T-ACE assessments during pregnancy have been noted to be more useful than medical records in determining PAE (Montag, 2016) and have demonstrated the ability to predict neurobehavioral effects in children that are associated with PAE when the cut-off mark is changed to 3 points instead of the usual 2-point cut-off (Chiodo et al., 2010). The T-ACE has been 


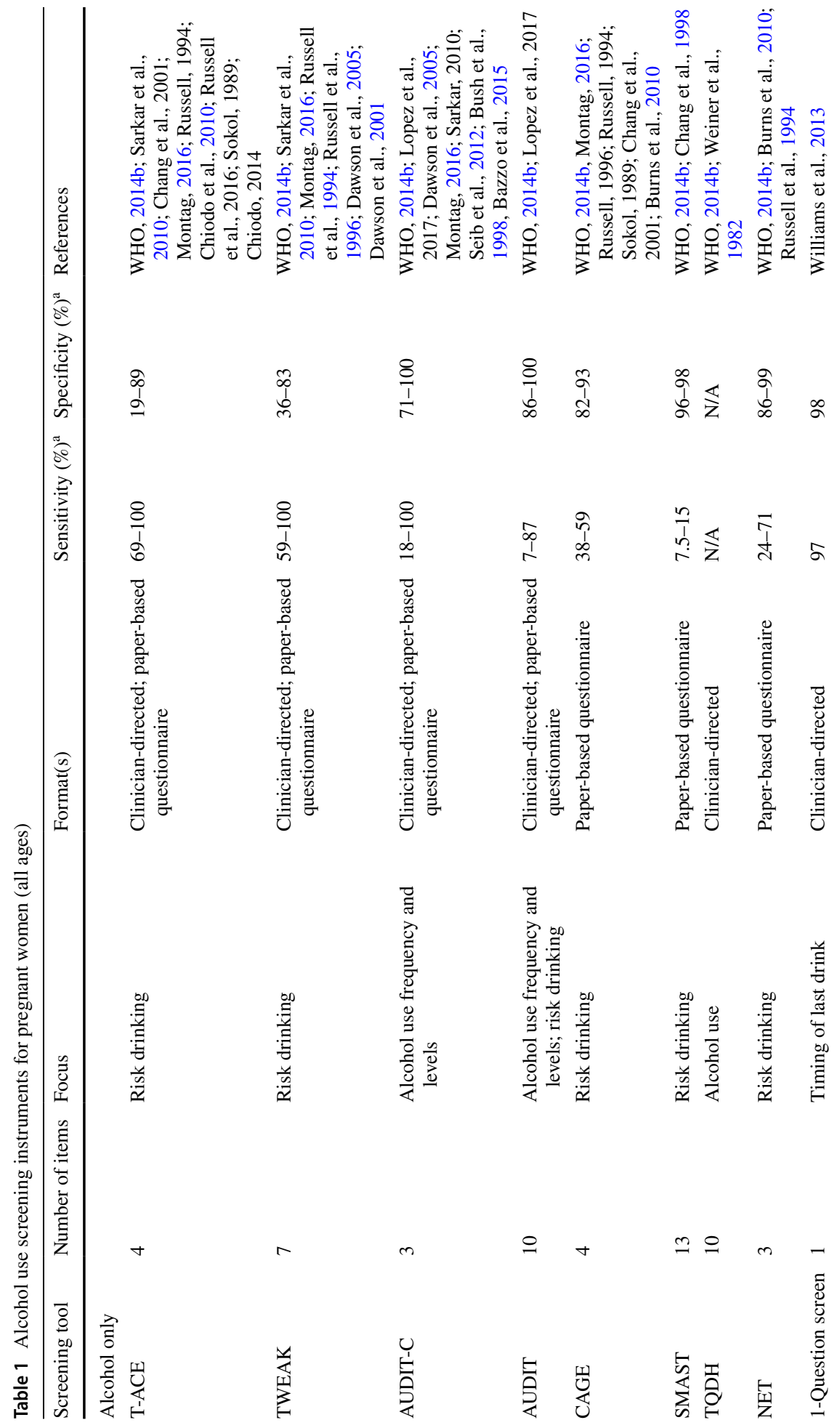




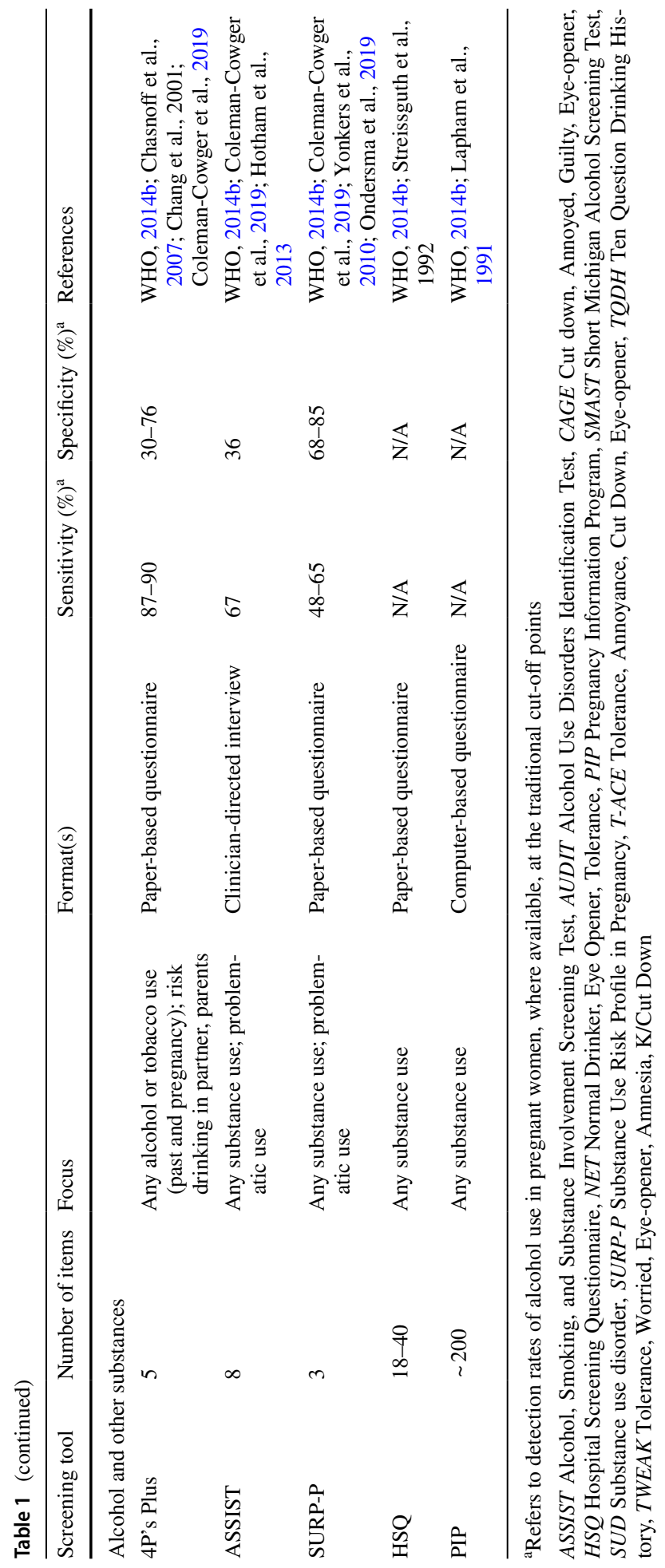


shown to be more sensitive and specific than the Short Michigan Alcohol Screening Test (SMAST) and Alcohol Use Disorders Identification Test (AUDIT) with respect to identifying lifetime alcohol diagnosis, risk drinking, as well as current drinking (Chang et al., 1998).

The TWEAK is an effective five-item screening tool used to identify women who are at-risk drinkers and has been validated in a number of sub-populations, including individuals with alcohol use disorder (AUD) in treatment, patients in an outpatient setting, and the general population (Chan et al., 1993). There have been mixed results regarding the use of the TWEAK screening tool in different countries. In Denmark, the TWEAK was found to be useful when identifying high-risk drinking during pregnancy (Praestegaard et al., 2018). When screening for alcohol use disorder (AUD) among pregnant women in Argentina, the TWEAK was found to have low reliability and did not perform as well as the AUDIT in this setting (Lopez et al., 2017). Similarly, the reliability of the TWEAK was found to be higher when used in the USA as compared to Argentina or Mexico, though it had lower reliability than the AUDIT in all 3 countries (Cremonte et al., 2010).

The T-ACE and TWEAK are optimized for heavy drinking, and use of these tests alone may produce a screening strategy that is not as sensitive as the test properties when it comes to detecting individuals with lower levels of alcohol consumption during pregnancy (Chang et al., 2001; Montag, 2016). Between the T-ACE and TWEAK, the T-ACE was found to be more sensitive among special sub-populations of pregnant women (Montag, 2016). Notably, in a high SES sub-population, the TWEAK and T-ACE were found to have similarly high sensitivity (99-100\% and 93-100\%, respectively), but poor specificity (36-43\% and 19-34\%, respectively), when identifying problem drinking in a pregnant population (Sarkar et al., 2010). It has also been suggested that both the T-ACE and TWEAK may perform better at cut-off points of 3 (Sarkar et al., 2010) or when the threshold for risk drinking is redefined (Chang et al., 2001), and this improves clinical utility of these instruments in identifying PAE (Montag, 2016; Chang et al., 2001). Nonetheless, both the T-ACE and the TWEAK screening instruments are considered to be the most effective screening tools for risk drinking in pregnant women in most countries (Lichtenberger et al., 2016; Seo et al., 2016).

The AUDIT-C includes a total of 3 items that focus on very heavy alcohol exposure (Montag, 2016), which are taken from the 10-item AUDIT. The AUDIT-C captures alcohol use frequency and quantity (Freeman et al., 2019), though the items alone do not distinguish time points of alcohol use with respect to pre-pregnancy, pregnancy recognition, and during pregnancy time periods (Poole et al., 2019). The AUDIT-C has been validated for use in pregnant women (Graves et al., 2020; Chiodo et al., 2010; Lopez et al., 2017; Freeman et al., 2019; Montag, 2016) and is even recommended as a means to confirm PAE in the FASD diagnostic assessment using Australian diagnostic guidelines in the general population (Freeman et al., 2019). Though the AUDIT-C was validated for use in pregnant women, it has been found to be unreliable in assessing alcohol use during pregnancy in an obstetric setting in Italy (Bazzo et al., 2015) and other countries (Montag, 2016). If a score of 5 points or over is obtained with the administration of the AUDIT-C, the clinician is directed to administer the remaining 7 items of the AUDIT, which measure risk drinking behaviors and features of alcohol use disorders. The AUDIT is a commonly used tool to measure alcohol use behaviors in the general population (Poole et al., 2019) and has been evaluated in a number of cultural groups, settings, and countries (Burns et al., 2010; Seo et al., 2016). A recent meta-analyses has suggested, however, that the AUDIT may not generally perform as well in countries with low prevalence rates of AUDs, or in 
women (Lange et al., 2019), which needs to be taken into consideration if all 10 items of the AUDIT are to be administered in an obstetric setting.

The CAGE (Cut down, Annoyed, Guilty, Eye-opener) is a four-item questionnaire examining problematic alcohol use, which has mixed results for identifying alcohol misuse in sub-populations globally. It may be superior to the TWEAK and T-ACE in identifying heavy alcohol use during pregnancy among women in Portuguese-speaking populations (Moraes et al., 2005), while other studies suggest it may be less effective in comparison to the TWEAK and T-ACE in other settings (Burns et al., 2010; Montag, 2016; Sarkar et al., 2010). The Short Michigan Alcohol Screening Test (SMAST) is a 13-item paper-based questionnaire, which can be used to examine alcohol use during pregnancy and lifetime alcohol dependence (Selzer, 1975; Chang et al., 1998). When used specifically in pregnancy, the SMAST has $7.5 \%$ sensitivity (Chang et al., 1998), which would indicate that the majority of women consuming alcohol during pregnancy would be missed with the use of this instrument. Both the CAGE and SMAST were not originally developed for use in pregnant women (WHO, 2014b; Montag, 2016), and the SMAST has been noted to perform poorly in prenatal alcohol use screening (Burns et al., 2010). Additionally, the CAGE may be less sensitive in women who are non-Caucasian or who belong to minority or disadvantaged populations (Montag, 2016).

The NET (Normal Drinker, Eye Opener, Tolerance) is a 3-item questionnaire which was developed for use in pregnant women (Burns et al., 2010) and captures risk levels of drinking (Bottoms et al., 1989; Burns et al., 2010; Chasnoff et al., 2007). Using a cut-off point of one or greater, the NET has high sensitivity (71\%) and specificity (86\%) (Russell et al., 1994); however, with one question asking women if they consider themselves to be a normal drinker, this tool may be particularly susceptible to social desirability bias among women who are currently pregnant. Furthermore, it does not specifically ask about alcohol use consumption levels or frequency, which is necessary to assess for risk of FASD, which means this tool is limited in detecting PAE.

A recently developed tool, the "1-Question screen," can also be used to screen pregnant women for alcohol with only one clinician-directed question that assesses the timing of the last drink consumed. This questionnaire has been compared to the T-ACE and had a $94.7 \%$ rate of agreement (Williams et al., 2013). The 1-Question screen has the added benefit of saving clinicians' time, which makes this less burdensome when considered for implementation on a population level (Williams et al., 2013).

The 4P's Plus is a five-question screening tool that could be used to identify obstetrical patients at risk for alcohol, tobacco, and/or illicit drug use (WHO, 2014b) and capture exposure which occurred prior to pregnancy recognition, as well as any problematic alcohol and drug use among their parents and partner (Chasnoff et al., 2005). The 4P's Plus screening tool has been validated in a low SES population in the USA and was found to have a sensitivity of $87 \%$ and specificity of $76 \%$ and reasonable reliability of $62 \%$ for a screening measure (Chasnoff et al., 2007). Furthermore, the 4P's Plus screening tool is able to identify women who were consuming alcohol but were not considered "heavy drinkers" (Chasnoff et al., 2007), making this particularly useful in identifying FASD risk.

Several additional instruments are available to assess alcohol and other substance use in pregnancy, which include the ASSIST, the HSQ, the PIP, and SURP-P (WHO, 2014b) (see Table 1). With regard to detecting any substance use (not limited to alcohol), the sensitivity is fairly high for the 4P's Plus (90.2\%), ASSIST (79.7\%), and SURP-P (92.4\%) (ColemanCowger et al., 2019). While the ASSIST has high specificity (82.8\%), the specificity of the 4P's Plus and SURP-P is lower in comparison (29.6\% and 21.8\%, respectively) (ColemanCowger et al., 2019). Between these 3 instruments, clinicians tend to prefer the 4P's Plus 
to detect any substance use in pregnant women, as it is brief and easy to administer (Trocin et al., 2020). In comparison, the HSQ and the PIP are far more time-intensive, as these questionnaires have 18-40 items and 200 items, respectively (WHO, 2014b).

In terms of screening specifically for alcohol use during pregnancy, the evidence is mixed overall for the effectiveness of each of the identified instruments used across countries (WHO, 2014b; Sarkar et al., 2010; Lopez et al., 2017; Bazzo et al., 2015; Burns et al., 2010). The utility of the alcohol use screening tool is dependent on the alcohol use behavior that is being measured (i.e., risk drinking or any alcohol consumption). Prenatal care providers may choose to screen for risk drinking based on previous maternal self-report of alcohol consumption during pregnancy. Alternatively, care providers can immediately screen for any levels of alcohol use with the AUDIT-C or the 4Ps Plus instruments. The administration of any of the identified screening instruments (Table 1) would not require clinician training (WHO, 2014b) and would take less than $15 \mathrm{~min}$ to administer (Burns et al., 2010).

All countries with published obstetric guidelines on alcohol use in pregnancy indicate that the best option for women is to completely abstain from alcohol during pregnancy, including guidelines in Australia, Canada, Scotland, Sweden, Switzerland, the UK, and the USA (Furtwaengler et al., 2013; Whitehall et al., 2007; Graves et al., 2020; ACOG, 2011). Various practitioner guidelines may also recommend approaches to alcohol use screening with available instruments. In the USA, the American College of Obstetricians and Gynecologists (ACOG) recommend that all women seeking obstetric-gynecologic care should be screened for alcohol use yearly and within the first trimester of pregnancy (ACOG, 2011). The ACOG suggests that routine screening be done using simple and validated tools such as the T-ACE with additional questions that capture both quantity and frequency (ACOG, 2011). The US Preventive Services Task Force (USPSTF) found that the full AUDIT, the abbreviated AUDIT-C, and single-question screening had the best performance with respect to detecting alcohol misuse in adults, young adults, and pregnant women; therefore, the US Preventive Services Task Force (USPSTF) prefer these screening instruments (Moyer et al., 2013).

With respect to adolescents, the American Academy of Pediatrics (AAP) recommends that pediatricians screen all adolescent patients for alcohol used with a screening tool that has been validated in adolescents, such as the CRAFFT (Car, Relax, Alone, Friends/ Family, Forget, Trouble), NIAAA Youth Alcohol Screen, or Brief Screener for tobacco, alcohol, and other drugs (BSTAD) (AAP, 2015). Though these tools are not specifically validated for use in pregnant adolescent girls, they are useful in identifying substance use issues in a sub-population at heightened risk of unplanned pregnancies and therefore alcohol-exposed pregnancies (AAP, 2015).

Within Canada, the Society of Obstetricians and Gynecologists of Canada (SOGC) recommends a structured approach to screening (Graves et al., 2020). The first level of screening involves approaches that are practice-based including the single question method, motivational interviewing, and supportive dialogue (Graves et al., 2020), which are the basis for the majority of data on alcohol use behavior available in Canadian birth registries (Poole et al., 2019). The second level of screening includes recommended screening instruments, such as the AUDIT-C or T-ACE for adults or the CRAFFT for adolescent populations (Graves et al., 2020). The use of other evidence-based tools, such as the TWEAK or CAGE, is also suggested, with the recommendation to pair all administered screening tools with access to brief interventions for alcohol use during pregnancy (Graves et al., 2020). 
The World Health Organization (WHO) recommends that healthcare providers discuss both past and present alcohol and substance use with all pregnant women as early in the pregnancy as possible and at every prenatal visit (WHO, 2014b).

\section{Level 3. Laboratory Screening Tools for PAE}

Maternal self-report will generally always underestimate alcohol consumption during pregnancy (Lange et al., 2014). The third level of alcohol use screening is the use of laboratorybased screening tools used to measure PAE via ethanol biomarkers (e.g., fatty acid ethyl esters) found in blood, maternal and neonatal hair, placenta, cord blood, and meconium. The decision to screen at this level, however, may be indicative of previously identified alcohol use risk behavior using other measures, including maternal self-report. One of the major drawbacks of this method is its being significantly more resource intensive as compared to routine screening based on self-report.

There are several ethanol blood biomarkers, which can be extracted from neonatal cord blood or maternal blood samples. Based on a narrative synthesis of PAE screening measures, it was found that using blood biomarkers alone had lower sensitivity and specificity and were less useful in assessing low to moderate levels of alcohol use during pregnancy, as compared to maternal self-report (Howlett et al., 2017). Generally, the testing of blood and urine samples is ineffective in detecting PAE overall, as it only captures recent alcohol use relative to the time of the sample (Joya et al., 2012). Similarly, the use of a breathalyzer administered during prenatal care visits was identified as an effective means to detect and quantify alcohol use during pregnancy in all 3 trimesters, among women in the Republic of Congo (Greenmyer et al., 2020a), but this would only capture alcohol use levels at the time of the prenatal care encounter.

The testing of meconium, or an infant's first few bowel movements, is considered the gold standard for assessing PAE. Meconium testing produces estimates that are 4 times higher as compared to self-report (Lange et al., 2014); however, meconium testing only captures PAE which occurred in late stages of pregnancy and likely identifies continuous drinking through pregnancy and therefore may miss many cases of PAE, especially pregnancies with early, light exposure. In addition to meconium testing, the analysis of maternal or neonatal hair is commonly used to establish PAE (Chan et al., 2004; Joya et al., 2012). There are several emerging technologies for additional biomarkers of PAE, including ethyl glucuronide, which has high sensitivity (82\%) and specificity (75\%) (Himes et al., 2015), as well as neonatal dried blood spots (Bakhireva et al., 2012) and placental tissue (Matlow et al., 2012; Shukla et al., 2011), for which the clinical utility is yet to be established.

\section{Improving Detection and Prevention of PAE}

Practitioner adherence to obstetric guidelines for alcohol use screening may vary across countries due to several factors. For example, numerous studies have identified time constraints or competing priorities of prenatal care providers as being a barrier to screening for alcohol use among pregnant women during visits (Wangberg et al., 2015; Chiodo et al., 2010; France et al., 2010; Doi et al., 2014; Doi et al., 2015). Qualitative research has also identified the lack of training among primary care providers as a barrier to engaging in 
conversations with pregnant women about their alcohol use (Wangberg et al., 2015; Chiodo et al., 2019; Taylor et al., 2007; Wouldes et al., 2009). Furthermore, healthcare providers may be deterred from screening for alcohol use based on the perceived lack of an available, validated screening tool and feelings of general discomfort in screening women (Oni et al., 2020). Improved training would be an important step to improve the PAE and FASD knowledge base and skills in screening among prenatal care providers including physicians, obstetricians, gynecologists, and midwives. Such training is also crucial for prenatal care providers involved in programs serving pregnant and postpartum women, such as the "Women, Infant and Child" (WIC) program, a widely utilized US federal program that provides supplemental foods, healthcare referrals, and nutrition education for low-income pregnant, breastfeeding, and non-breastfeeding postpartum women (Richards et al., 2010).

Recognition and medical confirmation of pregnancy may occur later for some women, at which point there may have already been significant levels of PAE. This may be influenced by unplanned pregnancies, which constitute a major roadblock in preventing alcohol use during pregnancy. An estimated $44 \%$ of pregnancies worldwide are unplanned, with rates even higher (up to 65\%) in developing countries (Bearak et al., 2018). Pregnancy planning may also affect the timing of first access to prenatal care, where prenatal care providers can screen and identify alcohol use patterns during the first and subsequent encounters. Notably, in Canada, an alcohol-exposed pregnancy was found to be associated with lesser odds of an optimally timed prenatal ultrasound compared to pregnancies that were not alcohol-exposed (OR: 0.69; 95\% CI: 0.53-0.90) (Abdullah et al., 2019). Furthermore, women experiencing violence (Cha et al., 2014; Jamieson et al., 2020) or those with mental health (Kim et al., 2005; Krans et al., 2013) or addiction issues (Roberts et al., 2010a) may have a delayed onset of utilization of prenatal care or may have limited access to prenatal care, especially if living in rural or remote communities (Simkhada et al., 2008). As these characteristics are also associated with an increased risk of alcohol use during pregnancy (Skagerstrom et al., 2011), this means that prenatal care providers may miss the opportunity to identify and address alcohol use during pregnancy among women who may be more prone to having alcohol-exposed pregnancies or which patients have a heightened need for access to brief interventions. It is also important for clinicians to recognize that PAE and FASD are typically recurrent in families and the provision of effective interventions for women at risk can prevent PAE in subsequent pregnancies and thus prevent future cases of FASD.

Research demonstrates that pre-pregnancy alcohol use patterns can significantly predict alcohol use during pregnancy; therefore, it is very important in screening for PAE (Skagerstrom et al., 2011; Montag, 2016; McQuire et al., 2020). Furthermore, alcohol use behaviors during pregnancy may be influenced by pre-existing beliefs and knowledge regarding the teratogenic effects of alcohol and FASD awareness (Nilsen et al., 2008; Watt et al., 2016; Williams et al., 2011). Globally, it may be viewed as more socially acceptable to drink alcohol during pregnancy in some countries, especially if healthcare providers themselves are not trained in the field of FASD. In countries with large-scale efforts to address FASD, however, there may be greater awareness of the harms of alcohol use during pregnancy, which may create stigma and underreporting of such behavior if it does occur (Watt et al., 2016). Stigma impacts maternal self-report in this case, even if prenatal care providers do sensitive and thorough screening for alcohol use during pregnancy. There is a significant level of stigma around substance use during pregnancy in general, which may deter pregnant women from reporting their consumption (Roberts et al., 2010a). 
In addition to stigma, alcohol use screening may have important legal implications in some countries, depending on laws related to substance use during pregnancy and prenatal injuries/maltreatment, which may also impact maternal self-report (Denison et al., 2014; Stone et al., 2015). Alcohol use screening measures, such as meconium testing, may be linked to the involvement of child protective services and temporary or permanent custody loss in some cases (Motherisk Commission, 2018) and therefore should be exercised with extreme precision and caution. The issue of child apprehension is related to health equity, which is demonstrated by the over-representation of certain sub-populations of women with children in the child welfare systems in North America (Denison et al., 2014; OHRC, 2018; Allan et al., 2015). These systems have been criticized for being discriminatory against Indigenous and Black communities (OHRC, 2018; Allan et al., 2015). This is especially important to note in the practice of alcohol use screening as this may be related to the over-screening of certain sub-populations in the prenatal care setting, while the general population in these communities may be simultaneously under-screened (Greenmyer et al., 2020b). Furthermore, fears of child apprehension may lead to limited utilization of healthcare (Denison et al., 2014) and underreporting of alcohol use during pregnancy. This, in turn, may result in underutilization of substance use disorder treatment, and the lack of screening may exclude women from access to brief interventions on alcohol use during pregnancy.

To minimize maternal underreporting of alcohol use in pregnancy, prenatal care providers can build rapport with their clients as they assess for alcohol use at multiple timepoints throughout the pregnancy, as this will increase the validity of maternal selfreport (WHO, 2014b; Doi et al., 2014). While underreporting may still occur, there is great value in screening by simply asking pregnant women about alcohol use, as this has been proven to result in greater awareness or altering of alcohol use behavior in pregnancy (Floyd et al., 2007; Tzilos et al., 2011). Furthermore, studies on pregnant women's perspectives show that screening for the use of alcohol is found to be acceptable during pregnancy (Roberts et al., 2010b; Toquinto et al., 2020). It is important for prenatal care providers to maximize the trust relationship, ensure a judgment-free environment, and conduct at least the first level of screening for pregnant women using alcohol and other substances.

The benefit of screening pregnant women for alcohol use is the capacity to facilitate access to brief interventions for women with identified alcohol-exposed pregnancies, to anticipate deliveries with increased complexity, and to identify newborns with increased risk for placement in the NICU, increased mortality risk, and those in need of developmental support or supervision. Evidence suggests brief interventions, such as educational, motivational interviewing or cognitive-behavioral interventions, are effective in reducing alcohol intake or rates of abstention from alcohol during pregnancy when led by healthcare providers and community leaders, in individual and group settings, including computer-delivered formats (O'Connor et al., 2018). Such interventions must be designed and implemented in light of the context of PAE among women that may participate in them, including pregnancy history, intergenerational trauma and discrimination, or child apprehension (Denison et al., 2014; Stone et al., 2015; OHRC, 2018; Allan \& Smykie, 2015).

Pregnant women deemed at risk can be referred to tailored interventions such as the parent-child assistance programs (PCAP), for example, which counsel pregnant women at risk of alcohol and drug-exposed pregnancies (Rasmussen et al., 2012; Thanh et al., 2015). This PCAP model has been proven to be cost-effective and effective in preventing cases of FASD in children among mothers at high-risk of alcohol exposed pregnancies, 
including mothers with FASD (Thanh et al., 2015). The PCAP model has been adopted in numerous locations in North America (Thanh et al., 2015) and is one example of an intervention that addresses the context of alcohol use during pregnancy for the targeted sub-population in a supportive manner (Pei et al., 2019). Another example is a very low cost, in-office pictorial intervention program for alcohol use in pregnancy that was developed and tested in the same communities in the Congo, which continues to be studied as a model for sub-Saharan countries (Williams et al., 2014). Furthermore, participation in programs such as the WIC in the USA can increase utilization of prenatal care among women at risk of alcohol-exposed pregnancies (Richards et al., 2010), which indirectly facilitates the detection and prevention of PAE.

\section{Limitations}

The current narrative review provides important implications for clinical practice, which must be understood within several existing limitations. First, the current review did not use specific inclusion or exclusion criteria for studies on current screening practices and tools. Second, the findings in this review are limited to the studies with data on current provider practices in high-income countries, which may not be an accurate depiction of screening practices globally. Additionally, there may be other effective screening strategies or tools currently being pilot-tested for which there is insufficient data or data that are yet to be published.

\section{Conclusions and Recommendations}

PAE screening stands to greatly benefit all women who consume alcohol prior to pregnancy, and it is one of the most important public health initiatives to prevent FASD. As such, alcohol screening should be mandatory and implemented consistently among all prenatal care providers. All prenatal care providers, including physicians, obstetricians, midwives, and nurses, must be trained in FASD prevention and must have the capacity to inform and advise pregnant women about the risks of alcohol use in pregnancy.

Prenatal care providers must carefully choose the alcohol use screening level based on individualized needs, such as the cultural context, pre-pregnancy alcohol use patterns, health status history, and the resources required to implement the screening measure. The evidence is mixed regarding the implementation of the first level of screening, with great variations across countries and provider types. If simply asking about alcohol use in pregnancy has the potential to change alcohol use behavior (Floyd et al., 2007; Tzilos et al., 2011), it is imperative that prenatal care providers take this opportunity. As patterns of alcohol use in the post-partum period tend to return to pre-pregnancy levels (Leggat et al., 2021), care providers can also use screening as a time to inform and advise women about the risks of alcohol use during breastfeeding, including impaired cognitive development of the infant (Gibson et al., 2018). Furthermore, consistent PAE screening will result in better data in population-based birth registries, which will allow researchers to measure the true prevalence of PAE and to design, implement, and evaluate PAE interventions over time.

There are also several evidence-based alcohol use screening instruments that have been validated as a means to screen for PAE: the AUDIT-C, 1-Question screen, and 4Ps Plus 
that measure any alcohol use and instruments such as the TWEAK, T-ACE, and CAGE which measure problematic use and risk drinking. Further research must be conducted to validate other existing screening instruments in pregnant women, in various cultural groups and settings. Prenatal care providers can choose from the recommended instruments based on their respective practitioner guidelines, while keeping in mind that any level or type of alcohol use in pregnancy can be harmful and therefore should be detected. All alcohol use screening of pregnant women must be done with care and precision and in a non-judgmental way, as these results may have important implications for child health.

Women who are at risk for, or have reported, alcohol-exposed pregnancies should be referred for a diagnostic assessment and further specialist treatment if needed or offered a brief intervention aimed at achieving alcohol abstention or, at the very least, reducing alcohol intake as much as possible. It is important to facilitate the design and implementation of appropriate interventions for women at risk of alcohol-exposed pregnancies. Prenatal care providers must be knowledgeable about the existing interventions in their communities that can offer supportive services to promote healthy behaviors into the postpartum period.

It is crucial for prenatal and other healthcare providers to provide a clear, evidencebased message for women about the risks of alcohol use in pregnancy, including FASD. As pre-pregnancy levels and beliefs about alcohol use can influence alcohol consumption in pregnancy, care providers must actively refute any common misconceptions, such as the idea that one drink per day is not known to be harmful. Providers should encourage all women to avoid consuming any alcohol throughout their pregnancy and while trying to become pregnant, emphasizing the benefits of doing so to the health and well-being of the mother and child.

Author Contribution DD conducted the literature review and narrative synthesis and drafted, reviewed, and revised the manuscript. LB contributed to the literature review and assisted in the interpretation of findings. SP supervised the development of the conception of this study and assisted in the interpretation of findings. All authors (DD, LB, and SP) have critically reviewed the manuscript for important intellectual content, have read and approved the final manuscript as it has been submitted, and agreed to be accountable for all aspects of the work.

Data Availability Not applicable.

\section{Declarations}

Ethics Approval Not applicable.

Consent Not applicable.

Competing Interests The authors declare no competing interests.

\section{References}

Abdullah, P., Landy, C. K., McCague, H., Macpherson, A., \& Tamim, H. (2019). Factors associated with the timing of the first prenatal ultrasound in Canada. BMC Pregnancy and Childbirth, 19(1), 164-164.

Allan, B. \& Smylie, J. (2015). First Peoples, second class treatment: The role of racism in the health and well-being of Indigenous peoples in Canada. Available from: https:/www.wellesleyinstitute.com/wpcontent/uploads/2015/02/Summary-First-Peoples-Second-Class-Treatment-Final.pdf. 
American Academy of Pediatrics. (2015) Substance use screening and implementation guide. Available from: https://www.aap.org/enus/Documents/substance_use_screening_implementation.pdf

American College of Obstetricians and Gynecologists Committee on Health Care for Underserved Women. (2011). Committee opinion no. 496: At-risk drinking and alcohol dependence: Obstetric and gynecologic implications. Obstetrics \& Gynecology, 118 (2), 383-8.

Arnold, K., Burke, M., Decker, A., Herzberg, E., Maher, M., \& Motz, K. (2013). Fetal alcohol spectrum disorders: Knowledge and screening practices of university hospital medical students and residents. Journal of Population Therapeutics and Clinical Pharmacology, 20(1), e18-25.

Bailey, B. A., \& Sokol, R. J. (2011). Prenatal alcohol exposure and miscarriage, stillbirth, preterm delivery, and sudden infant death syndrome. Alcohol Research \& Health: The Journal of the National Institute on Alcohol Abuse and Alcoholism, 34(1), 86-91.

Bakhireva, L. N., Sharkis, J., Shrestha, S., Miranda-Sohrabji, T., Williams, S., \& Miranda, R. C. (2017). Prevalence of prenatal alcohol exposure in the state of Texas as assessed by phosphatidylethanol in newborn dried blood spot specimens. Alcoholism, Clinical and Experimental Research, 41(5), 1004-1011.

Bazzo, S., Battistella, G., Riscica, P., Moino, G., Dal Pozzo, G., Bottarel, M., \& Czerwinsky, L. (2015). Reliability of a self-report Italian version of the AUDIT-C questionnaire, used to estimate alcohol consumption by pregnant women in an obstetric setting. Rivista Di Psichiatria, 50(2), 89-94.

Bearak, J., Popinchalk, A., Alkema, L., \& Sedgh, G. (2018). Global, regional, and subregional trends in unintended pregnancy and its outcomes from 1990 to 2014: Estimates from a Bayesian hierarchical model. The Lancet Global Health, 6(4), e380-e389.

Bottoms, S. F., Martier, S. S., Sokol, R. J. (1989) Refinements in screening for risk drinking in reproductive-aged women: The "NET" results. Alcoholism: Clinical and Experimental Research, 13:339.

Brownell, M., Enns, J. E., Hanlon-Dearman, A., Chateau, D., Phillips-Beck, W., Singal, D., et al. Health, social, education, and justice outcomes of Manitoba First Nations children diagnosed with fetal alcohol spectrum disorder: A population-based cohort study of linked administrative data. Canadian Journal of Psychiatry, 64(9), 611-620.

Burd, L., \& Popova, S. (2019). Fetal alcohol spectrum disorders: Fixing our aim to aim for the fix. International Journal of Environmental Research and Public Health, 16(20), 3978.

Burns, E., Gray, R., \& Smith, L. A. (2010). Brief screening questionnaires to identify problem drinking during pregnancy: A systematic review. Addiction (abingdon, England), 105(4), 601-614.

Bush, K., Kivlahan, D. R., McDonell, M. B., Fihn, S. D., \& Bradley, K. A. (1998). The AUDIT alcohol consumption questions (AUDIT-C): An effective brief screening test for problem drinking. Archives of Internal Medicine, 158(16), 1789-1795.

Cha, S., \& Masho, S. W. (2014). Intimate partner violence and utilization of prenatal care in the United States. Journal of Interpersonal Violence, 29(5), 911-927.

Chambers, C. C., Kable, J., Akshoomoff, N., Xu, R., Zellner, J., Honerkamp-Smith, G. et al. (2019). Fetal alcohol spectrum disorders in a pacific southwest city: Maternal and child characteristics. Alcoholism: Clinical \& Experimental Research, 43, 2578-2590.

Chan, D., Caprara, D., Blanchette, P., Klein, K., \& Koren, G. (2004). Recent developments in meconium and hair testing methods for the confirmation of gestational exposures to alcohol and tobacco smoke. Clin Biochemistry, 37, 429-438.

Chan, A.W.K., Pristach, E.A., Welte, J.W. and Russell, M. (1993). Use of the TWEAK test in screening for alcoholism/heavy drinking in three populations. Alcoholism: Clinical \& Experimental Research, 17, 1188-1192.

Chang, G., Wilkins-Haug, L., Berman, S., Goetz, M. A., Behr, H., \& Hiley, A. (1998). Alcohol use and pregnancy: Improving identification. Obstetrics and Gynecology, 91(6), 892-898.

Chang, G. (2001). Alcohol-screening instruments for pregnant women. Alcohol Research \& Health: The Journal of the National Institute on Alcohol Abuse and Alcoholism, 25(3), 204-209.

Chasnoff, I., McGourty, R., Bailey, G., et al. (2005). The 4P's Plus@ Screen for substance use in pregnancy: Clinical application and outcomes. Journal of Perinatology, 25, 368-374.

Chasnoff, I. J., Wells, A. M., McGourty, R. F., \& Bailey, L. K. (2007). Validation of the 4P's Plus screen for substance use in pregnancy validation of the 4P's Plus. Journal of Perinatology, 27(12), $744-748$.

Chiodo, L.M., Cosmian, C., Pereira, K., Kent, N., Sokol, R.J., Hannigan, J.H. (2019). Prenatal alcohol screening during pregnancy by midwives and nurses. Alcoholism: Clinical \& Experimental Research, 43(8), 1747-1758.

Chiodo, L. M., Delaney-Black, V., Sokol, R. J., Janisse, J., Pardo, Y., Hannigan, J. H. (2014). Increased cut-point of the TACER-3 screen reduces false positives without losing sensitivity in predicting 
risk alcohol drinking in pregnancy. Alcoholism: Clinical \& Experimental Research , 38(5), 1401-1408

Chiodo, L. M., Sokol, R. J., Delaney-Black, V., Janisse, J., \& Hannigan, J. H. (2010). Validity of the T-ACE in pregnancy in predicting child outcome and risk drinking. Alcohol (Fayetteville, N.Y.), 44(7), 595-603.

Coleman-Cowger, V. H., Oga, E. A., Peters, E. N., Trocin, K. E., Koszowski, B., \& Mark, K. (2019). Accuracy of three screening tools for prenatal substance use. Obstetrics \& Gynecology, 133(5), 952-961.

Cremonte, M., Ledesma, R. D., Cherpitel, C. J., \& Borges, G. (2010). Psychometric properties of alcohol screening tests in the emergency department in Argentina, Mexico, and the United States. Addictive Behaviors, 35(9), 818-825.

Dawson, D. A., Das, A., Faden, V. B., Bhaskar, B., Krulewitch, C. J., Wesley, B. (2001). Screening for high and moderate-risk drinking during pregnancy: A comparison of several TWEAK-based screeners. Alcoholism: Clinical \& Experimental Research, 25(9), 1342-1349.

Dawson, D. A., Grant, B. F., Stinson, F. S., Zhou, Y. (2005). Effectiveness of the derived Alcohol Use Disorders Identification Test (AUDIT-C) in screening for alcohol use disorders and risk drinking in the US general population. Alcoholism: Clinical \& Experimental Research, 29, 844-54.

Denison, J., Varcoe, C., \& Browne, A. J. (2014). Aboriginal women's experiences of accessing health care when state apprehension of children is being threatened. Journal of Advanced Nursing, 70(5), 1105-1116.

Doi, L., Cheyne, H., Jepson, R. (2014). Alcohol brief interventions in Scottish antenatal care: A qualitative study of midwives' attitudes and practices. BMC Pregnancy and Childbirth, 14(170).

Doi, L., Jepson, R., \& Cheyne, H. (2015). A realist evaluation of an antenatal programme to change drinking behaviour of pregnant women. Midwifery, 31(10), 965-972.

Floyd, R. L., Sobell, M., Velasquez, M. M., Ingersoll, K., Nettleman, M., Sobell, L., et al. (2007). Preventing alcohol-exposed pregnancies: A randomized controlled trial. American Journal of Preventive Medicine, 32(1), 1-10. https://doi.org/10.1016/j.amepre.2006.08.028

France, F., Henley, N., Payne, J., et al. (2010). Health professionals addressing alcohol use with pregnant women in Western Australia: Barriers and strategies for communication. Substance Use \& Misuse, 45(10), 1474-1490. https://doi.org/10.3109/10826081003682172

Fraser, S. L., Muckle, G., Abdous, B. B., Jacobson, J. L., \& Jacobson, S. W. (2012). Effects of binge drinking on infant growth and development in an Inuit sample. Alcohol (Fayetteville, N.Y.), 46(3), 277283. https://doi.org/10.1016/j.alcohol.2011.09.028

Freeman, J., Condon, C., Hamilton, S., Mutch, R. C., Bower, C., \& Watkins, R. E. (2019). Challenges in accurately assessing prenatal alcohol exposure in a study of fetal alcohol spectrum disorder in a youth detention center. Alcoholism, Clinical and Experimental Research, 43(2), 309-316.

Furtwaengler, N. A. F. F., \& de Visser, R. O. (2013). Lack of international consensus in low-risk drinking guidelines. Drug and Alcohol Review, 32(1), 11-18.

Gibson, L., \& Porter, M. (2018). Drinking or smoking while breastfeeding and later cognition in children. Pediatrics, 142(2), e20174266. https://doi.org/10.1542/peds.2017-4266

Graves, L., Carson, G., Poole, N., Patel, T., Bigalky, J., Green, R. C., Cook, J. L. (2020). Guideline No. 405: Screening and counselling for alcohol consumption during pregnancy. Journal of Obstetrics and Gynaecology Canada, 42(9), 1158-1173

Greenmyer, J. R., Klug, M. G., Kambeitz, C., Popova, S., \& Burd, L. (2018). A multi-country updated assessment of the economic impact of fetal alcohol spectrum disorder: Costs for children and adults. Journal of Addiction Medicine, 12(6), 466-473.

Greenmyer, J. R., Klug, M. G., Nkodia, G., Popova, S., Hart, B., \& Burd, L. (2020a). High prevalence of prenatal alcohol exposure detected by breathalyzer in the republic of the Congo, Africa. Neurotoxicology and Teratology, 80, 106892-106892.

Greenmyer, J. R., Popova, S., Klug, M. G., \& Burd, L. (2019). Fetal alcohol spectrum disorder: A systematic review of the cost of and savings from prevention in the United States and Canada. Addiction (abingdon, England), 115(3), 409-417.

Greenmyer, J. R., Stacy, J. M., Klug, M. G., Foster, K., Tiongson, C., \& Burd, L. (2020). Pregnancy status is associated with screening for alcohol and other substance use in the emergency department. Journal of Addiction Medicine, 14(4), e64-e69.

Henriksen, T. B., Hjollund, N. H., Jensen, T. K., Bonde, J. P., Andersson, A. M., Kolstad, H., et al. (2004). Alcohol consumption at the time of conception and spontaneous abortion. American Journal of Epidemiology, 160, 1-7.

Himes, S. K., Dukes, K. A., Tripp, T., Petersen, J. M., Raffo, C., Burd, L., . . . for the Prenatal Alcohol in SIDS and Stillbirth (PASS) Network. (2015). Clinical sensitivity and specificity of meconium fatty 
acid ethyl ester, ethyl glucuronide, and ethyl sulfate for detecting maternal drinking during pregnancy. Clinical Chemistry (Baltimore, Md.), 61(3), 523-532.

Hotham, E., Ali, R., White, J., Sullivan, T., Robinson, J. (2013). Investigation of the Alcohol, Smoking, and Substance Involvement Screening Test (the ASSIST) Version 3.0 in Pregnancy. Addictive Disorders \& Their Treatment, 12(3), 123-135. doi: https://doi.org/10.1097/ADT.0b013e3182636904

Howlett, H., Abernethy, S., Brown, N. W., Rankin, J., \& Gray, W. K. (2017). How strong is the evidence for using blood biomarkers alone to screen for alcohol consumption during pregnancy? A systematic review. European Journal of Obstetrics \& Gynecology and Reproductive Biology, 213, 45-52.

Jamieson, B. (2020). Exposure to interpersonal violence during pregnancy and its association with women's prenatal care utilization: A meta-analytic review. Trauma, Violence, \& Abuse, 21(5), 904-921.

Jones, K. L., Smith, D. W., Ulleland, C. N., \& Streissguth, A. P. (1973). Pattern of malformation in offspring of chronic alcoholic mothers. The Lancet, 1(7815), 1267-1271.

Jones, K. L., \& Smith, D. W. (1973). Recognition of the fetal alcohol syndrome in early infancy. The Lancet, 302(7836), 999-1001.

Joya, X., Friguls, B., Ortigosa, S., Papaseit, E., Martínez, S. E., Manich, A., et al. (2012). Determination of maternal-fetal biomarkers of prenatal exposure to ethanol: A review. Journal of Pharmaceutical and Biomedical Analysis, 69, 209-222. https://doi.org/10.1016/j.jpba.2012.01.006

Kim, H. G., Mandell, M., Crandall, C., Kuskowski, M. A., Dieperink, B., \& Buchberger, R. L. (2005). Antenatal psychiatric illness and adequacy of prenatal care in an ethnically diverse inner-city obstetric population. Archives of Women's Mental Health, 9(2), 103-107.

Krans, E. E., Krans, E. E., Davis, M. M., Davis, M. M., Palladino, C. L., \& Palladino, C. L. (2013). Disparate patterns of prenatal care utilization stratified by medical and psychosocial risk. Maternal and Child Health Journal, 17(4), 639-645.

Lange, S., Shield, K., Koren, G., Rehm, J., \& Popova, S. (2014). A comparison of the prevalence of prenatal alcohol exposure obtained via maternal self-reports versus meconium testing: A systematic literature review and meta-analysis. BMC Pregnancy and Childbirth, 14(1), 127-127.

Lange, S., Shield, K., Monteiro, M., \& Rehm, J. (2019). Facilitating screening and brief interventions in primary care: A systematic review and meta-analysis of the AUDIT as an indicator of alcohol use disorders. Alcoholism, Clinical and Experimental Research, 43(10), 2028-2037.

Lapham, S. C., Kring, M. K., \& Skipper, B. (1991). Prenatal behavioral risk screening by computer in a health maintenance organization-based prenatal care clinic. American Journal of Obstetrics and Gynecology, 165(3), 506-514.

Lebel, C., Roussotte, F., \& Sowell, E. R. (2011). Imaging the impact of prenatal alcohol exposure on the structure of the developing human brain. Neuropsychology Review, 21(2), 102-118.

Leggat, G., Livingston, M., Kuntsche, S., \& Callinan, S. (2021). Changes in alcohol consumption during pregnancy and over the transition towards parenthood. Drug and Alcohol Dependence, 225, 108745. https://doi.org/10.1016/j.drugalcdep.2021.108745

Lemola, S., Gkiouleka, A., Urfer-Maurer, N., Grob, A., Tritten Schwarz, K., \& Meyer-Leu, Y. (2020). Midwives' engagement in smoking- and alcohol-prevention in prenatal care before and after the introduction of practice guidelines in Switzerland: Comparison of survey findings from 2008 and 2018. BMC Pregnancy and Childbirth, 20(1), 31.

Lichtenberger, A., Conde, K. N., \& Cremonte, M. (2016). Investigación en intervención breve y consumo de alcohol durante la gestación: Productividad e impacto. Revista Chilena De Obstetricia y Ginecologia, 81(1), 56-62.

López, M., Lichtenberger, A., Conde, K., \& Cremonte, M. (2017). Psychometric properties of brief screening tests for alcohol use disorders during pregnancy in Argentina. Revista Brasileira De Ginecologia e Obstetrícia, 39(7), 322-329.

Matlow, J. N., Aleksa, K., Lubetsky, A., \& Koren, G. (2012). The detection and quantification of ethyl glucuronide in placental tissue and placental perfusate by headspace solid-phase microextraction coupled with gas chromatography-mass spectrometry. Journal of Population Therapeutics and Clinical Pharmacology, 19, e473-e482.

May, P. A., Blankenship, J., Marais, A., Gossage, J. P., Kalberg, W. O., Joubert, B., \& Seedat, S. (2013). Maternal alcohol consumption producing fetal alcohol spectrum disorders (FASD): Quantity, frequency, and timing of drinking. Drug and Alcohol Dependence, 133(2), 502-512. https://doi.org/10. 1016/j.drugalcdep.2013.07.013

McQuire, C., Daniel, R., Hurt, L., Kemp, A., \& Paranjothy, S. (2020). The causal web of foetal alcohol spectrum disorders: A review and causal diagram. European Child \& Adolescent Psychiatry, 29(5), 575-594. https://doi.org/10.1007/s00787-018-1264-3

Montag, A. C. (2016). Fetal alcohol-spectrum disorders: Identifying at-risk mothers. International Journal of Women's Health, 8, 311-323. 
Moraes, C. L., Viellas, E. F., \& Reichenheim, M. E. (2005). Assessing alcohol misuse during pregnancy: Evaluating psychometric properties of the CAGE, T-ACE and TWEAK in a Brazilian setting. Journal of Studies on Alcohol, 66(2), 165-173.

Motherisk Commission. (2018). Harmful impacts: The reliance on hair testing in child protection. A report of the Motherisk Commission. Available from: https://www.attorneygeneral.jus.gov.on.ca/english/ about/pubs/motherisk/.

Moyer, V. A., \& Preventive Services Task Force (2013). Screening and behavioral counseling interventions in primary care to reduce alcohol misuse: U.S. preventive services task force recommendation statement. Annals of internal medicine, 159(3), 210-218. https://doi.org/10.7326/0003-4819-159-3-20130 8060-00652

Nilsen, P., Holmqvist, M., Hultgren, E., Bendtsen, P., \& Cedergren, M. (2008). Alcohol use before and during pregnancy and factors influencing change among Swedish women. Acta Obstetricia Et Gynecologica Scandinavica, 87(7), 768-774.

Nykjaer, C., Alwan, N. A., Greenwood, D. C., Simpson, N. A. B., Hay, A. W. M., White, K. L. M., et al. (2014). Maternal alcohol intake prior to and during pregnancy and risk of adverse birth outcomes: Evidence from a British cohort. Journal of Epidemiology \& Community Health, 68, 542-549.

O’Connor, E. A., Perdue, L. A., Senger, C. A., Rushkin, M., Patnode, C. D., Bean, S. I., et al. (2018). Screening and behavioral counseling interventions to reduce unhealthy alcohol use in adolescents and adults: Updated evidence report and systematic review for the US preventive services task force. JAMA: The Journal of the American Medical Association, 320, 1910-1928.

O’Connor, M. J., Kogan, N., Findlay, R. (2002). Prenatal alcohol exposure and attachment behavior in children. Alcoholism: Clinical \& Experimental Research,26(10), 1592-602.

Oh, S. S., Kim, Y. J., Kim, Y. J., Jang, S., Park, S., Nam, C. M., \& Park, E. C. (2020). Hospitalizations and mortality among patients with fetal alcohol spectrum disorders: A prospective study. Scientific Reports, 10(1), 19512-19512.

Ondersma, S. J., Chang, G., Blake-Lamb, T., Gilstad-Hayden, K., Orav, J., Beatty, J. R., \& Yonkers, K. A. (2019). Accuracy of five self-report screening instruments for substance use in pregnancy. Addiction (abingdon, England), 114(9), 1683-1693.

Oni, H. T., Buultjens, M., Blandthorn, J., Davis, D., Abdel-Latif, M., Islam, M. M. (2020). Barriers and facilitators in antenatal settings to screening and referral of pregnant women who use alcohol or other drugs: A qualitative study of midwives' experience. Midwifery, 81, 102595.

Ontario Human Rights Commission. Interrupted childhoods: Over-representation of Indigenous and Black children in Ontario child welfare [Internet]. Report 978-1-4868-1117-5. Ottawa, ON; 2018 [cited 2020 Dec 06]. Available from: http://www.ohrc.on.ca/en/interrupted-childhoods.

Patra, J., Bakker, R., Irving, H., Jaddoe, V. W., Malini, S., \& Rehm J. (2011). Dose-response relationship between alcohol consumption before and during pregnancy and the risks of low birthweight, preterm birth and small for gestational age (SGA)—A systematic review and meta-analyses. BJOG: International Journal of Obstetrics \& Gynaecology, 118(12), 1411-1421.

Payne, J., Elliott, E., D’Antoine, H., O’Leary, C., Mahony, A., Haan, E., \& Bower, C. (2005). Health professionals' knowledge, practice and opinions about fetal alcohol syndrome and alcohol consumption in pregnancy. Australian and New Zealand Journal of Public Health, 29(6), 558-564. https://doi.org/10. 1111/j.1467-842x.2005.tb00251.x

Pei, J., Carlson, E., Tremblay, M., \& Poth, C. (2019). Exploring the contributions and suitability of relational and community-centered fetal alcohol spectrum disorder (FASD) prevention work in first nation communities. Birth Defects Research, 111(12), 835-847.

Pollard, M. S., Tucker, J. S., \& Green, H. D. (2020). Changes in adult alcohol use and consequences during the COVID-19 pandemic in the US. JAMA Network Open, 3(9), e2022942-e2022942.

Poole, N., Schmidt, R. A., Bocking, A., Bergeron, J., \& Fortier, I. (2019). The potential for fetal alcohol spectrum disorder prevention of a harmonized approach to data collection about alcohol use in pregnancy cohort studies. International Journal of Environmental Research and Public Health, 16(11), 2019.

Popova, S., Lange, S., Burd, L., \& Rehm, J. (2016a). The economic burden of fetal alcohol spectrum disorder in Canada in 2013. Alcohol and Alcoholism (oxford, Oxfordshire), 51(3), 367-375. https://doi.org/ 10.1093/alcalc/agv117

Popova, S., Lange, S., Probst, C., Gmel, G., \& Rehm, J. (2017). Estimation of national, regional, and global prevalence of alcohol use during pregnancy and fetal alcohol syndrome: A systematic review and meta-analysis. The Lancet Global Health, 5(3), e290-e299.

Popova, S., Lange, S., Probst, C., Parunashvili, N., \& Rehm, J. (2017). Prevalence of alcohol consumption during pregnancy and fetal alcohol spectrum disorders among the general and aboriginal populations in Canada and the United States. European Journal of Medical Genetics, 60(1), 32-48. 
Popova, S., Lange, S., Shield, K., Burd, L., \& Rehm, J. (2019). Prevalence of fetal alcohol spectrum disorder among special subpopulations: A systematic review and meta-analysis. Addiction, 114(7), 1150-1172.

Popova, S., Lange, S., Shield, K., Mihic, A., Chudley, A. E., Mukherjee, R. A., et al. (2016). Comorbidity of fetal alcohol spectrum disorder: A systematic review and meta-analysis. The Lancet, 387(10022), 978-987.

Præstegaard, C., Kesmodel, P. S., \& Kesmodel, U. S. (2018). Is TWEAK a valid screening questionnaire to identify alcohol risk drinkers among pregnant women in Denmark? Acta Obstetricia Et Gynecologica Scandinavica, 97(4), 483-490.

Rasmussen, C., Kully-Martens, K., Denys, K., Badry, D., Henneveld, D., \& Wyper, K. (2012). The effectiveness of a community-based intervention program for women at-risk for giving birth to a child with fetal alcohol spectrum disorder (FASD). Community Mental Health Journal, 48(1), 12-21.

Rehm, J., Kilian, C., Ferreira-Borges, C., Jernigan, D., Monteiro, M., Parry, C. D. H., \& Manthey, J. (2020). Alcohol use in times of the COVID 19: Implications for monitoring and policy. Drug and Alcohol Review, 39(4), 301-304.

Richards, R., Merrill, R. M., Baksh, L., \& McGarry, J. (2010). Maternal health behaviors and infant health outcomes among homeless mothers: U.S. special supplemental nutrition program for women, infants, and children (WIC) 2000-2007. Preventive Medicine, 52(1), 87-94.

Roberts, S. C. M., \& Nuru-Jeter, A. (2010). Women's perspectives on screening for alcohol and drug use in prenatal care. Women's Health Issues, 20(3), 193-200.

Roberts, S. C. M., \& Pies, C. (2010). Complex calculations: How drug use during pregnancy becomes a barrier to prenatal care. Maternal and Child Health Journal, 15(3), 333-341.

Russell, M., Martier, S. S., Sokol, R. J., et al. (1994). Screening for pregnancy risk-drinking. Alcoholism: Clinical \& Experimental Research, 18(5), 1156-1161.

Russell, M., Martier, S. S., Sokol, R. J., Mudar, P., Jacobson, S., \& Jacobson, J. (1996). Detecting risk drinking during pregnancy: A comparison of four screening questionnaires. American Journal of Public Health, 86(10), 1435-1439.

Sarkar, M., Einarson, T., \& Koren, G. (2010). Comparing the effectiveness of TWEAK and T-ACE in determining problem drinkers in pregnancy. Alcohol and Alcoholism (oxford, Oxfordshire), 45(4), 356-360. https://doi.org/10.1093/alcalc/agq022

Scholder, S., Wehby, G. L., Lewis, S., \& Zuccolo, L. (2014). Alcohol exposure in utero and child academic achievement. The Econonic Journal, 124(576), 634-667.

Seib, C. A., Daglish, M., Heath, R., Booker, C., Reid, C., \& Fraser, J. (2012). Screening for alcohol and drug use in pregnancy. Midwifery, 28(6), 760-764.

Selzer, M. L., Vinokur, A., \& van Rooijen, L. (1975). A self-administered Short Michigan Alcoholism Screening Test (SMAST). Journal of Studies on Alcohol and Drugs, 36(1), 117-126. https://doi. org/10.15288/jsa.1975.36.117

Seo, Y. R., Kim, J. S., Kim, S. S., Yoon, S. J., Suh, W. Y., \& Youn, K. (2016). Development of a simple tool for identifying alcohol use disorder in female Korean drinkers from previous questionnaires. Korean Journal of Family Medicine., 37(1), 18-24.

Sher, J. (2020). Fetal alcohol spectrum disorders: Preventing collateral damage from COVID-19. The Lancet. Public Health, 5(8), e424-e424.

Shukla, P. K., Sittig, L. J., Ullmann, T. M., Redei, E. E. (2011). Candidate placental biomarkers for intrauterine alcohol exposure. Alcoholism: Clinical \& Experimental Research. 2011, 35, 559-565.

Simkhada, B., Teijlingen, E. R. V., Porter, M., \& Simkhada, P. (2008). Factors affecting the utilization of antenatal care in developing countries: Systematic review of the literature. Journal of Advanced Nursing, 61(3), 244-260. https://doi.org/10.1111/j.1365-2648.2007.04532.x

Skagerstróm, J., Chang, G., \& Nilsen, P. (2011). Predictors of drinking during pregnancy: A systematic review. Journal of Women's Health (Larchmont, N.Y.2002), 20(6), 901-913.

Sokol, R. J., Delaney-Black, V., Nordstrom, B. (2003). Fetal alcohol spectrum disorder. JAMA: Journal of the American Medical Association, 290:2996-2999.

Sokol, R. J., Martier, S. S., \& Ager, J. W. (1989). The T-ACE questions: Practical prenatal detection of risk-drinking. American Journal of Obstetrics \& Gynecology, 160(4), 863-870.

Stone, R. (2015). Pregnant women and substance use: Fear, stigma, and barriers to care. Health \& Justice, 3, 2. https://doi.org/10.1186/s40352-015-0015-5

Streissguth, A. P., \& Giunta, C. T. (1992). Subject recruitment and retention for longitudinal research: Practical considerations for a nonintervention model. In M. M. Kilbey \& K. Asghar (Eds.), Methodological Issues in Epidemiological, Prevention, and Treatment Research on Drug-Exposed Women and Their Children Rockville: NIDA Monograph No. 117 (137-154) US DHHS Public Health Services. 
Taylor, P., Zaichkin, J., Pilkey, D., Leconte, J., Johnson, B. K., \& Peterson, A. C. (2007). Prenatal screening for substance use and violence: Findings from physician focus groups. Maternal and Child Health Journal, 11(3), 241-247. https://doi.org/10.1007/s10995-006-0169-9

Thanh, N. X., Jonsson, E., Moffatt, J., Dennett, L., Chuck, A. W., \& Birchard, S. (2015). An economic evaluation of the parent-child assistance program for preventing fetal alcohol spectrum disorder in Alberta, Canada. Administration and Policy in Mental Health, 42(1), 10-18. https://doi.org/10. 1007/s10488-014-0537-5

Toquinto, S. M., Berglas, N. F., McLemore, M. R., Delgado, A., \& Roberts, S. C. M. (2020). Pregnant women's acceptability of alcohol, tobacco, and drug use screening and willingness to disclose use in prenatal care. Women's Health Issues, 30(5), 345-352.

Trocin, K. E., Weinstein, N. I., Oga, E. A., Mark, K. S., \& Coleman-Cowger, V. (2020). Prenatal practice staff perceptions of three substance use screening tools for pregnant women. Journal of Addiction Medicine, 14(2), 139-144.

Tzilos, G. K., Sokol, R. J., \& Ondersma, S. J. (2011). A randomized phase 1 trial of a brief computerdelivered intervention for alcohol use during pregnancy. Journal of Women's Health, 20, 1517-1524.

Wangberg, S. C. (2015). Norwegian midwives' use of screening for and brief interventions on alcohol use in pregnancy. Sexual \& Reproductive Healthcare : Official Journal of the Swedish Association of Midwives, 6(3), 186-190. https://doi.org/10.1016/j.srhc.2015.03.001

Watt, M. H., Eaton, L. A., Dennis, A. C., Choi, K. W., Kalichman, S. C., Skinner, D., \& Sikkema, K. J. (2016). Alcohol use during pregnancy in a South African community: Reconciling knowledge, norms, and personal experience. Maternal and Child Health Journal, 20(1), 48-55. https://doi.org/10. 1007/s10995-015-1800-4

Weiner, L., Rosett, H. L., \& Edelin, K. C. (1982). Behavioral evaluation of fetal alcohol education for physicians. Alcoholism: Clinical \& Experimental Research, 6(2), 230-233.

Whitehall, J. S. (2007). National guidelines on alcohol use during pregnancy: A dissenting opinion. The Medical Journal of Australia, 186(1), 35-37. https://doi.org/10.5694/j.1326-5377.2007.tb00786.x

Williams, A., Nkombo, Y., Nkodia, G., Leonardson, G., Martsolf, K., \& Burd, L. (2014). Effectiveness of a novel low cost intervention to reduce prenatal alcohol exposure in the Congo. Open Journal of Pediatrics, 4(1), 84-92.

Williams, A. D., Nkombo, Y., Nkodia, G., Leonardson, G., \& Burd, L. (2013). Prenatal alcohol exposure in the republic of the Congo: Prevalence and screening strategies: Prenatal alcohol exposure in Congo. Birth Defects Research: A Clinical and Molecular Teratology, 97(7), 489-496.

Williams, L., Zapata, L. B., D’Angelo, D. V., Harrison, L., \& Morrow, B. (2011). Associations between preconception counseling and maternal behaviors before and during pregnancy. Maternal and Child Health Journal, 16(9), 1854-1861.

World Health Organization. (2018). Global status report on alcohol and health 2018. Geneva: World Health Organization. License: CC BY-NC-SA 3.0 IGO. Available from: https://apps.who.int/iris/bitstream/ handle/10665/274603/9789241565639-eng.pdf.

World Health Organization. (2014b). Guidelines for the identification and management of substance use and substance use disorders in pregnancy. Available from: https://apps.who.int/iris/bitstream/handle/ 10665/107130/9789241548731_eng.pdf;jsessionid=528480BDEF513AE9130A22E5EB1C182E? sequence $=1$

World Health Organization (2014a). WHO Global Action Plan for the Prevention and Control of NCDs 2013-2020 Available from: file:///C:/Users/16478/Downloads/9789241506236_eng\%20(1).pdf

Wouldes, T. (2009). What health professionals know \& do about alcohol and other drug use during pregnancy: A research report in collaboration with Alcohol Healthwatch. University of Auckland.

Yonkers, K. A., Gotman, N., Kershaw, T., Forray, A., Howell, H. B., \& Rounsaville, B. J. (2010). Screening for prenatal substance use: Development of the Substance Use Risk Profile-Pregnancy scale. Obstetrics and Gynecology, 116(4), 827-833. https://doi.org/10.1097/AOG.0b013e3181ed8290

Publisher's Note Springer Nature remains neutral with regard to jurisdictional claims in published maps and institutional affiliations. 